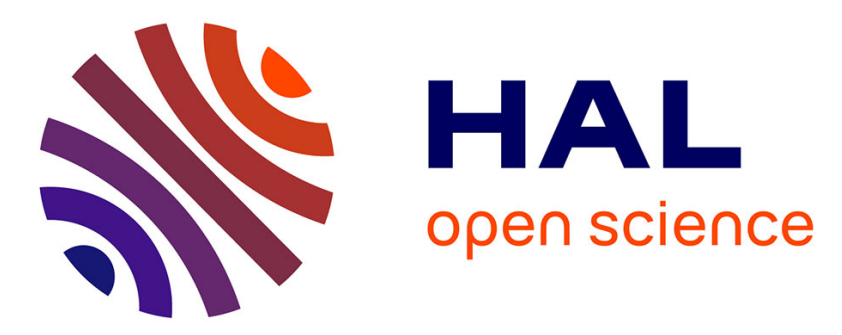

\title{
First Order Error Correction for Trimmed Quadrature in Isogeometric Analysis
}

\author{
Felix Scholz, Angelos Mantzaflaris, Bert Jüttler
}

\section{To cite this version:}

Felix Scholz, Angelos Mantzaflaris, Bert Jüttler. First Order Error Correction for Trimmed Quadrature in Isogeometric Analysis. Advanced Finite Element Methods with Applications, Springer, pp.297-321, 2019, 10.1007/978-3-030-14244-5_15 . hal-02197664

\section{HAL Id: hal-02197664 \\ https://hal.inria.fr/hal-02197664}

Submitted on 30 Jul 2019

HAL is a multi-disciplinary open access archive for the deposit and dissemination of scientific research documents, whether they are published or not. The documents may come from teaching and research institutions in France or abroad, or from public or private research centers.
L'archive ouverte pluridisciplinaire HAL, est destinée au dépôt et à la diffusion de documents scientifiques de niveau recherche, publiés ou non, émanant des établissements d'enseignement et de recherche français ou étrangers, des laboratoires publics ou privés. 


\title{
First order error correction for trimmed quadrature in isogeometric analysis
}

\author{
Felix Scholz, Angelos Mantzaflaris, and Bert Jüttler
}

\begin{abstract}
In this work, we develop a specialized quadrature rule for trimmed domains, where the trimming curve is given implicitly by a real-valued function on the whole domain. We follow an error correction approach: In a first step, we obtain an adaptive subdivision of the domain in such a way that each cell falls in a predefined base case. We then extend the classical approach of linear approximation of the trimming curve by adding an error correction term based on a Taylor expansion of the blending between the linearized implicit trimming curve and the original one. This approach leads to an accurate method which improves the convergence of the quadrature error by one order compared to piecewise linear approximation of the trimming curve. It is at the same time efficient, since essentially the computation of one extra one-dimensional integral on each trimmed cell is required. Finally, the method is easy to implement, since it only involves one additional line integral and refrains from any point inversion or optimization operations. The convergence is analyzed theoretically and numerical experiments confirm that the accuracy is improved without compromising the computational complexity.
\end{abstract}

\section{Introduction}

A common representation of a Computer-Aided Design (CAD) model is a boundary representation (B-rep), which typically consists of trimmed tensor-product NURBS patches. A trimmed surface patch consists of a tensor-product surface and a set of trimming curves on the surface that represent the boundary of the actual surface.

Felix Scholz

Radon Institute for Computational and Applied Mathematics, Austrian Academy of Sciences, Linz, Austria, e-mail: felix.scholz@ ricam.oeaw.ac.at

Angelos Mantzaflaris · Bert Jüttler

Institute of Applied Geometry, Johannes Kepler University Linz, Austria,

e-mail: angelos.mantzaflaris@jku.at, bert.juettler@jku.at 
Therefore, it represents only a part of the full tensor-product surface yet no explicit parametric representation is available. In this paper we are interested in applying numerical integration on a trimmed surface patch.

Computing integrals over trimmed domains both efficiently and accurately remains a challenging problem, notably for use in the frame of isogeometric analysis (IgA) [11]. The latter computational framework aims at a unification of the representations used in CAD and in numerical simulation, therefore operating directly on trimmed patches. The reader is referred to [19] for a recent review on trimming in $\mathrm{CAD}$ and $\mathrm{IgA}$. We remark that different CAD representations are possible, e.g. subdivision surface-based models or T-spline models, see [1,2, 12].

Multiple challenges arise for $\operatorname{IgA}$ on trimmed domains. One issue is the efficient coupling between adjacent trimmed patches. To this end, a finite cell method with weak coupling has been proposed in [24], a tearing and interconnecting approach was recently studied in [32] as well as Discontinuous Galerkin (DG) methods [9, $10,31]$. Another issue is the numerical stability of the trimmed basis functions, since basis functions with a tiny support can appear around the trimmed boundary. Several modified bases have been considered, such as immersed B-splines [25] and extended B-splines [18] to overcome the issue. Finally, the problem of applying numerical quadrature on a trimmed patch is a challenge in its own right.

One first approach to integrating over trimmed surfaces is to place quadrature points on the full surface and set the weights of the points lying outside the trimmed domain to zero. However, this method has no guarantees and the integration error cannot be controlled easily. In engineering practice local adaptive quadrature is used on top of it, that is, subdivision is performed around the trimmed region and quadrature nodes are placed in each sub-cell $[8,13,28]$. This approach can generate an extensive number of quadrature points, thus posing efficiency barriers.

Another approach is to perform a reparameterization (either globally, or locally at the element level), also known as "untrimming"; this puts more effort in the geometric side and results in tensor-product patches, which can be handled in an efficient way [27]. However, it is known that exact reparameterization is not feasible and approximate solutions result in cracks or overlaps in the model which require special treatment, e.g. by means of DG methods [9, 31]. In [14], the authors use base cases for the trimmed elements and perform local untrimming, using the intersection points of the trimmed curve and the boundary; see also [29] for some applications of this machinery in optimization. In [26] a local untrimming on the element level is performed by a projection method, which can be interpolation or least-squares fitting.

When the trimming curves are complicated and have high degree, it is typical to compute a piecewise linear approximation of the boundary to simplify further processing [1, 3, 15, 23]. However, when it comes to numerical integration, the geometry approximation error accumulates in the final result, and deteriorates the overall approximation order. Alternatively, in [20] the linearization is avoided, and a quadrature rule is constructed for each trimmed element by solving a momentfitting, non-linear system to obtain quadrature nodes and weights, which are all contained inside the domain. 
The problem of integrating over trimmed domains also arises in the context of geometrically unfitted finite element methods [4]. In these methods, the solution of a PDE is approximated on a background finite element mesh that is cut by the boundary of the computational domain. A method for quadrature in this context is presented in [16]. The author uses piecewise linear approximation of the boundary and applies a transformation of the finite element mesh in order to improve the approximation order. For the case of Cut Finite Element Methods (CutFEM, [7]), a method for numerical integration using boundary integrals and Taylor approximation in a Nitsche formulation was developed in [5].

In the present work we develop an efficient and accurate quadrature rule for the approximation of integrals over trimmed domains. The trimming curve is given implicitly by a real-valued level function on the whole domain. This does not impose any restrictions, since any trimming curve can be converted into this format by employing implicitization techniques, which can be either exact or approximate ones, see, e.g., $[6,30]$. Our method is based on an error correction approach. In a first step, we obtain an adaptive subdivision of the domain in such a way that each cell falls in a predefined base case. We then extend the classical approach of linear approximation of the trimming curve by adding an error correction term based on a Taylor expansion of the blending between the linearized implicit trimming curve and the original one.

In terms of accuracy, the method improves the local quadrature error in each cell by two orders of magnitude compared to the piecewise linear approximation of the trimming curve, thus providing an extra order of convergence globally. In particular, cubic order of convergence is achieved with a negligible additional computational cost. The efficiency of the method is achieved by the fact that it requires solely the evaluation of the trimming function at the vertices of the cells and the quadrature nodes, and refrains from any kind of point inversion or non-linear solving. Furthermore, our method is easy to implement, since the resulting nodes for the correction term are simply one-dimensional Gauss nodes and their corresponding weights are given by a direct computation. Moreover, we do not need to test for quadrature points outside the integration domain or treat them in a different way. Overall, it is straight-forward to upgrade existing codes to incorporate our method.

In the next section, we state the problem of trimmed quadrature with a implicitly defined trimming curve. In Section 3 we explain the first step of the method, the subdivision of the domain into quadrature cells belonging to certain base cases. Section 4 deals with the piecewise linear approximation of the trimming function which is then extended by the first order error correction in Section 5. We analyze the convergence behavior of our method theoretically and experimentally in Sections 6 and 7 , respectively. 


\section{Problem formulation}

Throughout this paper, we consider integrals of bivariate functions on trimmed domains. More precisely, we assume that a sufficiently smooth function

$$
f:[0,1]^{2} \rightarrow \mathbb{R}
$$

is given, which is defined on the entire unit square. In addition, we restrict the unit square by trimming with an implicitly defined curve $\tau(x, y)=0$, which is defined by another smooth bivariate function

$$
\tau:[0,1]^{2} \rightarrow \mathbb{R} .
$$

This results in the trimmed domain

$$
\Omega_{\tau}=\left\{(x, y) \in[0,1]^{2}: \tau(x, y) \geq 0\right\} .
$$

We seek for quadrature rules that provide approximate values of the integral

$$
I_{\tau} f=\int_{\Omega_{\tau}} f(x, y) \mathrm{d} y \mathrm{~d} x .
$$

These quadrature rules shall take the form

$$
Q_{\tau} f=\sum_{i} w_{i} f\left(x_{i}, y_{i}\right)
$$

with a finitely many quadrature nodes $\left(x_{i}, y_{i}\right)$ and associated weights $w_{i}$. Two comments about this problem are in order:

1. As described in the introduction, this problem originates in isogeometric analysis, where one needs to solve it in order to perform isogeometric discretizations of partial differential equations on trimmed patches. Typically, the function $f$ then takes the form

$$
f(x, y)=D B_{j}(x, y) \bar{D} B_{k}(x, y) K(x, y)
$$

where the functions $B_{i}$ are bivariate tensor-product B-splines or polynomial segments thereof and the kernel $K$ reflects the influence of the geometry mapping (i.e., the parameterization of the computational domain by a NURBS surface) and the coefficient functions of the PDE. In many situations, this results in a piecewise rational function $f$.

2. Usually, the trimming functions in CAD and not given implicity but by low degree parametric curves. It is then possible to convert these curves into implicit form by invoking suitable implicitization techniques, which can be either exact or approximate ones, see e.g. [6, 30]

Our approach to finding a quadrature rule consists of two steps. First we subdivide the domain to reach a certain discretization size, while simultaneously ensuring that 
we arrive at a sufficiently simple configuration on each cell. Second we evaluate the contribution of each cell to the total value of the integral. These steps will be discussed in the next three sections.

\section{Adaptive subdivision of the domain}

Given a step size $h$, we subdivide the domain uniformly until the cell size does not exceed $h$. Subsequently, we perform adaptive subdivision until each resulting cell $K$ is an instance of one of the five base cases depicted in Fig. 1. The resulting set of quadrature cells will be denoted by $\mathscr{K}$.

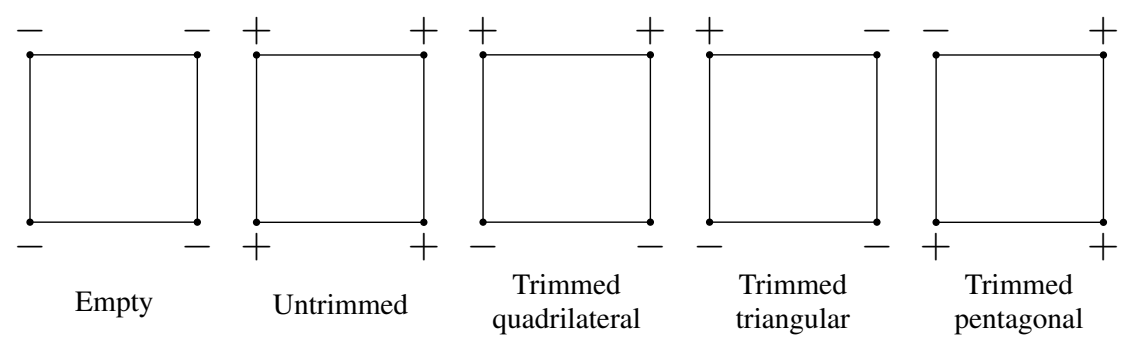

Fig. 1 Sign distributions of the trimming function $\tau$ for all five base cases (up to rotations).

More precisely, we use only evaluations of the trimming function at the cell vertices to identify the base cases, similar to the marching cubes algorithm [21]. Consequently, the method does not detect branches of the trimming curve that leave and re-enter the cell within the same edge. In order to illustrate this fact, Fig. 2 shows two instances of each trimmed base case.

Zero values at the vertices are treated as positive numbers. Consequently, there are only two sign distributions that do not represent a base case, see Fig. 3. These situations are dealt with by uniformly subdividing the corresponding cell. This process is guaranteed to terminate if no singularities of the trimming curve are present (which is always the case in practice).

We summarize our adaptive subdivision approach to the generation of quadrature cells $\mathscr{K}$ in the following algorithm. 


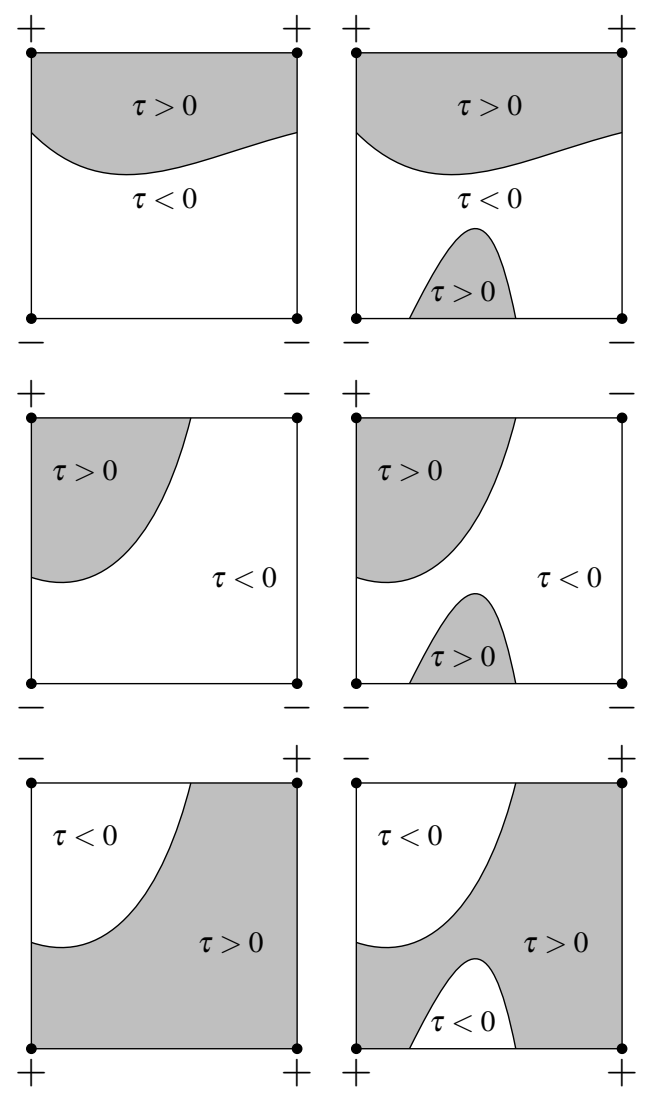

Fig. 2 Two instances of each trimmed base case (curved quadrilateral, triangle and pentagon).
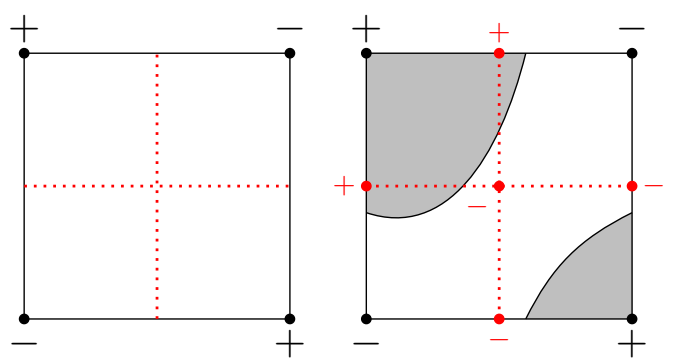

Fig. 3 Left: Sign distribution that does not represent a base case. Right: Uniform subdivision (quadsection) of this cell. 
- QuadratureCells, input: $\tau, h>0$

- Let the initial cell be $K=[0,1]^{2}$.

- Repeat for each untreated cell $K$ :

$$
\begin{aligned}
& \text { if } \operatorname{Size}(K)<h \text { and BaseCase }(\tau, K) \\
& \quad \operatorname{Report} K \\
& \text { else } \\
& \quad K_{1}, \ldots, K_{4}=\text { Quadsect }(K) \\
& \quad \text { Mark } K_{1}, \ldots, K_{4} \text { as untreated. }
\end{aligned}
$$

Fig. 4 shows an instance of quadrature cells generated by the algorithm. The zero set of the trimming function consists of two parallel lines, which are parallel to one of the square's diagonals. The parameter $h$ was chosen as $\frac{1}{2}$. For this specific instance of $\tau$, the algorithm needs one or two additional subdivision steps at the northwest and southeast corners of the domain.

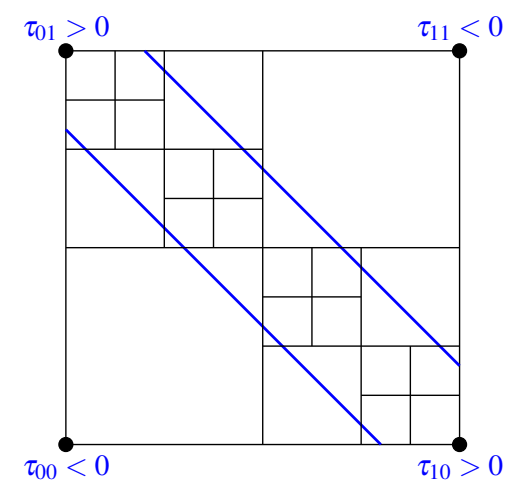

Fig. 4 Quadrature cells for $h=\frac{1}{2}$. The trimming curve is shown in blue.

\section{Linearized trimmed quadrature}

We perform the quadrature individually on each cell $K \in \mathscr{K}$. Thus, we need to approximate the integral

$$
\int_{K_{\tau}} f(x, y) \mathrm{d} y \mathrm{~d} x
$$

where

$$
K_{\tau}=\{(x, y) \in K: \tau(x, y)>0\} .
$$

This approximation is trivial for the first two base cases: The value of the integral equals zero in the first case, and it is approximated by a tensor-product Gauss rule in the second one. 
In order to perform this approximation in the remaining three base cases, we replace $\tau$ by another function $\sigma$. That function is chosen such that the integral

$$
\int_{K_{\sigma}} f(x, y) \mathrm{d} y \mathrm{~d} x
$$

over the region

$$
K_{\sigma}=\{(x, y) \in K: \sigma(x, y)>0\}
$$

enclosed by the zero-level set of $\sigma$ admits a simple evaluation. This is achieved by using a suitable linear approximation of $\tau$, that is, the level set $\sigma(x, y)=0$ is simply a straight line segment.

The evaluation of (9) using numerical quadrature is based on the intersections of $\sigma(x, y)=0$ with the boundary of the cell. We determine these intersections by linear interpolation of the function values of $\tau$ at the vertices of the cell. Fig. 5 shows examples for this approximation. For this choice of $\sigma$, the linearized integral (9) belongs to the same base case as the original integral (7).

For the quadrilateral case we proceed as follows: First, we construct a bilinear parameterization of $K_{\sigma}$. Second, we transform the integral to the associated parameter domain and evaluate its value using Gauss quadrature with $n$ evaluations per parametric direction, where $n$ is chosen by the user. The triangular case is dealt with analogously, by using a parameterization with a singularity at the involved cell vertex. In the pentagonal case, the identity

$$
K_{\sigma}=K \backslash K_{-\sigma}
$$

allows to evaluate (9) by combining results for the untrimmed and the triangular case.
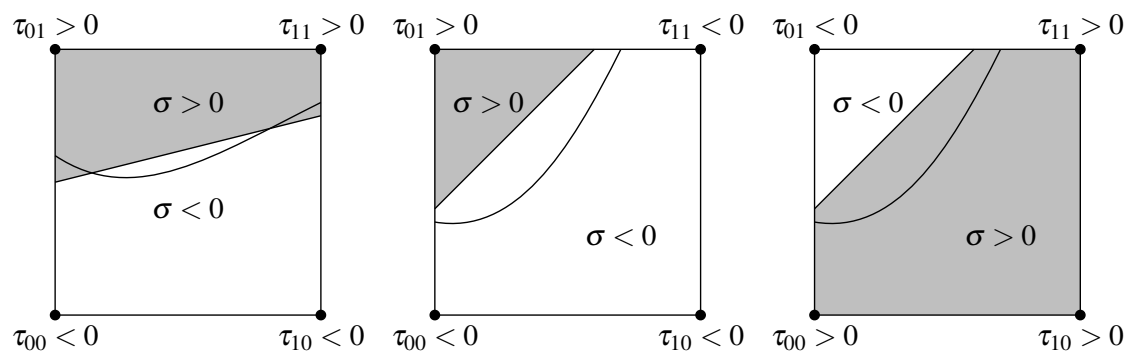

Fig. 5 A simple approximation of the three trimmed base cases.

The resulting linearized trimmed quadrature rule will be referred to as

$$
\mathrm{LT}(h, n),
$$

where $h$ is the maximum cell size and $n$ denotes the number of Gauss nodes. Clearly, the values generated by the LT rule converge to the true integral as $h$ is decreased. 
As we shall see later, however, the rather rough approximation of the quadrature domain limits the order of convergence.

\section{First order correction}

We improve the order of convergence of the LT rule by adding an error correction term for the last three base cases. This term is found by performing a Taylor expansion.

Throughout this section, we consider a fixed trimmed cell

$$
K=\left[x_{0}, x_{0}+h\right] \times\left[y_{0}, y_{0}+h\right] \in \mathscr{K}
$$

which is either a quadrangular or triangular base case, see Figure 5. Recall that the pentagonal case is solved by considering the complementary triangular domain.

Linear blending of the trimming function $\tau$ and its linear approximation $\sigma$ leads to the subsets

$$
K_{\sigma+u(\tau-\sigma)}=\{(x, y) \in K: \sigma(x, y)+u(\tau(x, y)-\sigma(x, y))>0\}, \quad u \in[0,1],
$$

that define the function

$$
F(u)=\int_{K_{\sigma+u(\tau-\sigma)}} f(x, y) \mathrm{d} y \mathrm{~d} x .
$$

It attains the exact value of (4) for $u=1$, while the LT rule is based on the approximate evaluation of $F(0)$. We improve the accuracy by adding a correction term that is based on the first two terms of the Taylor series

$$
F(1)=F(0)+F^{\prime}(0)+R_{1}(1)
$$

of $F$ around $u=0$, where $R_{1}$ denotes the remainder.

In order to compute $F^{\prime}(0)$, we observe that the level set of the function obtained by linear blending defines a function $y=c_{u}(x)$ or $x=c_{u}(y)$ for sufficiently small values of $u$, where the projection of the trimming curve onto the $x$ or $y$ axis specifies the domain

$$
\left[a_{u}, b_{u}\right]
$$

respectively. If both choices are possible, we choose the one with the larger domain for $u=0$.

Without loss of generality, we consider the first case

$$
\left[a_{u}, b_{u}\right] \subset\left[x_{0}, x_{0}+h\right]
$$

where the function satisfies

$$
\sigma\left(x, c_{u}(x)\right)+u\left(\tau\left(x, c_{u}(x)\right)-\sigma\left(x, c_{u}(x)\right)\right)=0,
$$




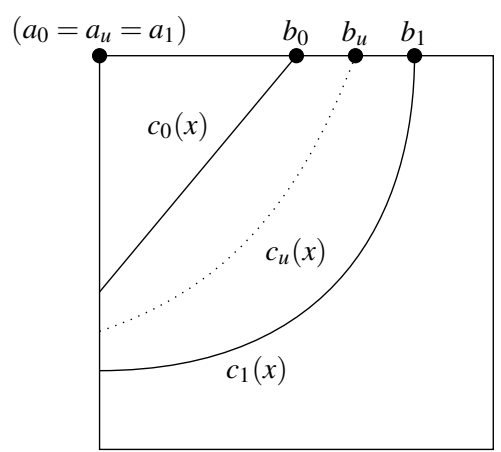

Fig. 6 Linear blending of $\tau$ and $\sigma$ in the triangular base case.

see Figure 6. By differentiating (19) we observe that

$$
\frac{\partial}{\partial u} c_{u}(x)=\frac{-\tau\left(x, c_{u}(x)\right)+\sigma\left(x, c_{u}(x)\right)}{\frac{\partial \sigma}{\partial y}\left(x, c_{u}(x)\right)+u\left(\frac{\partial \tau}{\partial y}\left(x, c_{u}(x)\right)-\frac{\partial \sigma}{\partial y}\left(x, c_{u}(x)\right)\right)} .
$$

The function $F$ in (15) can be rewritten as

$$
F(u)=\int_{a_{u}}^{b_{u}} \int_{c_{u}(x)}^{y_{0}+h} f(x, y) \mathrm{d} y \mathrm{~d} x .
$$

Its first derivative thus evaluates to

$$
\begin{aligned}
F^{\prime}(u)= & -\int_{a_{u}}^{b_{u}} f\left(x, c_{u}(x)\right) \frac{\partial}{\partial u} c_{u}(x) \mathrm{d} x \\
& -\frac{\mathrm{d}}{\mathrm{d} u} a_{u} \int_{c_{u}\left(a_{u}\right)}^{y_{0}+h} f\left(a_{u}, y\right) \mathrm{d} y+\frac{\mathrm{d}}{\mathrm{d} u} b_{u} \int_{c_{u}\left(b_{u}\right)}^{y_{0}+h} f\left(b_{u}, y\right) \mathrm{d} y
\end{aligned}
$$

The integration limit satisfies $b_{u}=x_{0}+h$ or

$$
c_{u}(b(u))=y_{0}+h .
$$

Consequently, the third term in (22) vanishes since either the integral or the factor in front of it take value zero. Similarly, the second term vanishes as well.

Finally we use (20), (22) and the fact that $\sigma$ vanishes on the graph of $c_{0}$,

$$
\sigma\left(x, c_{0}(x)\right)=0,
$$

to rewrite the first order correction term

$$
F^{\prime}(0)=\frac{1}{\frac{\partial}{\partial y} \sigma} \int_{a_{0}}^{b_{0}} f\left(x, c_{0}(x)\right) \tau\left(x, c_{0}(x)\right) \mathrm{d} x
$$


as a univariate integral over the linearized trimming curve. An approximate value is computed using a Gauss rule with $k$ quadrature nodes.

Note that we only used the zero level set of $\sigma$ in Section 5. The value of the correction term depends on the gradient of the linearized trimming function, which we did not discuss so far. In fact, we would like to choose $\nabla \sigma$, and more specifically $\frac{\partial \sigma}{\partial y}$, so that it approximates sufficiently well $\nabla \tau$. A good choice is to set $\frac{\partial \sigma}{\partial y}$ using finite differences over the values of $\tau$ at the cell's vertices. In the quadrilateral case we use the average

$$
\frac{\partial \sigma}{\partial y}=\frac{1}{2}\left(\frac{\tau_{01}-\tau_{00}}{h}+\frac{\tau_{11}-\tau_{10}}{h}\right)=\frac{\tau_{01}-\tau_{00}+\tau_{11}-\tau_{10}}{2 h}
$$

of the finite differences over the two edges that intersect the trimming curve. In the triangular case we use the finite difference

$$
\frac{\partial \sigma}{\partial y}=\frac{\tau_{01}-\tau_{00}}{h}
$$

over the edge which intersects the trimming curve.

The resulting corrected linearized trimmed quadrature rule (with first order correction term) will be referred to as

$$
\operatorname{CLT}(h, n, k),
$$

where $h$ is the maximum cell size, and $n$ resp. $k$ are the numbers of bivariate resp. univariate quadrature nodes. In addition, we use

$$
\operatorname{CLT}_{K}(h, n, k),
$$

to denote the value contributed by an individual cell $K \in \mathscr{K}$.

If the trimming function $\tau$ is linear and thus $\sigma=\tau$, then

$$
\tau\left(x, c_{0}(x)\right)=0 .
$$

Consequently, the correction term (25) vanishes. In this case, CLT and LT give equivalent results.

\section{Convergence result}

In this section we will show that the first order error correction in the CLT rule improves the convergence by one order with respect to the non-corrected LT rule. More precisely, we will prove this result for a slightly modified version of CLT, obtained by adapting the quadrature cells, which we denote as CLT ${ }^{\star}$. Intuitively, we construct the modified version of CLT as follows: We look at all cells where the gradient of the trimming function is "almost" horizontal or vertical, for some point. 
In this case we fuse two adjacent cells in such a way that the trimmed cell becomes of the quadilateral base case which is easier to treat theoretically than the triangular case.

First we prove two technical results about the local errors in the trimmed cells of the quadrilateral base case (Lemma 1) and of the triangular base case (Lemma 2). Second we combine them with the known approximation properties of the employed Gauss rules to estimate the global quadrature error in Theorem 1.

Both lemmas consider a rectangular cell (not necessarily a square) $K$ of size $h$ and a trimming function $\tau$ defined on it. We derive error bounds that applies to all cells of these base cases.

We say that the cell satisfies the assumptions (about the base cases) in the strong sense if the trimming curve crosses the boundary in exactly two points.

Lemma 1. Assume that a rectangular cell $K$ fulfills the assumptions of the quadrilateral base case in the strong sense, and the trimming function $\tau$ and its linear approximation $\sigma$ satisfy the inequalities

$$
\left|\frac{\partial \tau}{\partial y}(x, y)\right| \geq C_{1} \quad, \quad \forall(x, y) \in K
$$

and

$$
\begin{aligned}
\|\sigma-\tau\|_{L^{\infty}(K)} & \leq C_{2} h^{2}, \\
\|\nabla \sigma-\nabla \tau\|_{L^{\infty}(K)} & \leq C_{3} h
\end{aligned}
$$

for certain positive constants $C_{1}, C_{2}, C_{3}$. Then there exists a constant

$$
C_{\text {quad }}\left(C_{1}, C_{2}, C_{3}, f\right)
$$

which depends solely on these three constants and $f$, such that the corrected trimmed quadrature on this cell fulfills for $n=k=2$

$$
\left|I_{\tau, K} f-\operatorname{CLT}_{K}(h, 2,2) f\right| \leq C_{\text {quad }} h^{4} .
$$

Proof. We denote the rectangular cell by $K=\left[x_{0}, x_{0}+\alpha h\right] \times\left[y_{0}, y_{0}+\beta h\right]$ where $\left(x_{0}, y_{0}\right)$ is the lower left vertex and $\alpha, \beta>0$. Since by the monotonicity assumption (31) the trimming curve $\tau(x, y)=0$ can be written as a graph, the same is true for all intermediate curves if $h$ is sufficiently small. By differentiating (22) and using that both $a_{u}=x_{0}$ and $b_{u}=x_{0}+\alpha h$ are constant, we obtain, for all $u \in[0,1]$,

$$
F^{\prime \prime}(u)=-\int_{a_{u}}^{b_{u}} \frac{\partial}{\partial y} f\left(x, c_{u}(x)\right)\left(\frac{\partial}{\partial u} c_{u}(x)\right)^{2}+f\left(x, c_{u}(x)\right) \frac{\partial^{2}}{\partial u^{2}} c_{u}(x) \mathrm{d} x
$$

We observe that under the assumptions (31) - (33)

$$
\left|\frac{\partial}{\partial u} c_{u}(x)\right|=\frac{\left|\tau\left(x, c_{u}(x)\right)-\sigma\left(x, c_{u}(x)\right)\right|}{\left|(1-u) \frac{\partial}{\partial y} \sigma+u \frac{\partial}{\partial y} \tau\left(x, c_{u}(x)\right)\right|} \leq C^{\prime} h^{2} .
$$


where $C^{\prime}$ depends on $C_{1}, C_{2}, C_{3}$. By differentiating (19) twice we obtain

$$
\frac{\partial^{2}}{\partial u^{2}} c_{u}(x)=\frac{-2 \frac{\partial}{\partial u} c_{u}\left(\frac{\partial}{\partial y} \tau-\frac{\partial}{\partial y} \sigma\right)-u\left(\frac{\partial}{\partial u} c_{u}\right)^{2} \frac{\partial^{2}}{\partial y^{2}} \tau}{(1-u) \frac{\partial}{\partial y} \sigma+u \frac{\partial}{\partial y} \tau\left(x, c_{u}(x)\right)}
$$

and thus using again the assumptions on $\tau$ and $\sigma$ we get

$$
\left|\frac{\partial^{2}}{\partial u^{2}} c_{u}(x)\right| \leq C^{\prime \prime} h^{3}
$$

where $C^{\prime \prime}$ depends again on $C_{1}, C_{2}, C_{3}$. By estimating the integral by the supremum we conclude that for all $u \in[0,1]$

$$
F^{\prime \prime}(u) \leq C^{\prime \prime \prime} h^{4} .
$$

The result is obtained by combining Taylor's theorem with the approximation properties of the employed Gauss rules for the bi- and univariate quadrature.

Lemma 2. Assume that a rectangular cell $K$ satisfies the assumptions of the triangular base case (in the strong sense) and that in addition to the assumptions (31)-(33) in Lemma 1 there is a constant $C_{4}$ independent of $h$, such that

$$
\left|\frac{\partial \tau}{\partial x}(x, y)\right| \geq C_{4}>0 \quad, \quad \forall(x, y) \in K
$$

Then, there exists a constant $C_{\text {triangle }}\left(C_{1}, C_{2}, C_{3}, C_{4}, f\right)$, such that the corrected trimmed quadrature on this cell fulfills

$$
\left|I_{\tau, K} f-\operatorname{CLT}_{K}(h, 2,2) f\right| \leq C_{\text {triangle }} h^{4} .
$$

Proof. Again, we write $K=\left[x_{0}, x_{0}+\alpha h\right] \times\left[y_{0}, y_{0}+\beta h\right]$.. In the triangular case, $b_{u}$ in (22) is not constant but defined implicitly by

$$
c_{u}\left(b_{u}\right)=y_{0}+\beta h .
$$

For the second derivative of $F$ this means that an additional term appears which depends on the derivative of $b_{u}$ :

$$
\begin{aligned}
F^{\prime \prime}(u)= & -\int_{a_{u}}^{b_{u}} \frac{\partial}{\partial y} f\left(x, c_{u}(x)\right)\left(\frac{\partial}{\partial u} c_{u}(x)\right)^{2}+f\left(x, c_{u}(x)\right) \frac{\partial^{2}}{\partial u^{2}} c_{u}(x) \mathrm{d} x \\
& -f\left(b_{u}, c_{u}\left(b_{u}\right)\right) \frac{\partial}{\partial u} c_{u}\left(b_{u}\right) \frac{\mathrm{d}}{\mathrm{d} u} b_{u} .
\end{aligned}
$$

In order to estimate the last term in (44), we compute

$$
\frac{\mathrm{d}}{\mathrm{d} u} b_{u}=-\frac{\frac{\partial}{\partial u} c_{u}\left(b_{u}\right)}{\frac{\partial}{\partial x} c_{u}\left(b_{u}\right)} .
$$


Differentiating (19) with respect to $x$ leads to

$$
\frac{\partial}{\partial x} c_{u}(x)=-\frac{\frac{\partial}{\partial x} \sigma+u\left(\frac{\partial}{\partial x} \tau\left(x, c_{u}(x)\right)-\frac{\partial}{\partial x} \sigma\right)}{\frac{\partial}{\partial y} \sigma+u\left(\frac{\partial}{\partial y} \tau\left(x, c_{u}(x)\right)-\frac{\partial}{\partial y} \sigma\right)} .
$$

Using assumption (41) we conclude

$$
\left|\frac{\partial}{\partial x} c_{u}(x)\right| \geq C^{\prime \prime \prime \prime}>0
$$

and thus

$$
\left|\frac{\mathrm{d}}{\mathrm{d} u} b_{u}\right| \leq C^{\prime \prime \prime \prime \prime \prime} h^{2}
$$

Therefore, in view of (37) and (39) the last term in (36) satisfies

$$
f\left(b_{u}, c_{u}\left(b_{u}\right)\right) \frac{\partial}{\partial u} c_{u}\left(b_{u}\right) \frac{\mathrm{d}}{\mathrm{d} u} b_{u} \leq C^{\prime \prime \prime \prime \prime \prime \prime} h^{4}
$$

and the result follows.

Unfortunately, even with these two results at hand, we cannot analyze the quadrature rule CLT directly. This is due to two reasons: First, we cannot guarantee that all the triangular cells in the subdivision $\mathscr{K}$ satisfy the assumption of Lemma 2. Second, there may be trimmed cells which are not base cases in the strong sense. Both problems are resolved by suitably modifying the quadrature rule.

More precisely, we construct a modified quadrature rule CLT* ${ }^{*}$ by replacing the subdivision $\mathscr{K}$ of $\Omega$ with a new subdivision $\mathscr{K}^{\star}$. We begin by using creating a uniform grid of cells of size $\frac{h}{2}$. We assume that $h$ is small enough, such that all cells belong to one of the base cases. The subdivision $\mathscr{K}^{0}$ consists of all cells that possess a non-empty intersection with the integration domain. Note that this also includes cells where the trimming curve crosses the same edge twice.

We will obtain $\mathscr{K}^{\star}$ by merging some of the trimmed cells in $\mathscr{K}^{0}$.

First, we define the constants $C_{1}$ and $C_{4}$ that are to be used in Lemmas 1 and 2 as

$$
C_{1}=C_{4}=\frac{1}{4} \min _{\tau(x, y)=0}\|\nabla \tau(x, y)\|_{2}
$$

If $h$ is sufficiently small this means that each trimmed cell $K \in \mathscr{K}^{0}$ belongs to one of three classes:

1. Class $\mathrm{H}$ (horizontal gradient): For all $(x, y) \in K$ we have

$$
\left|\frac{\partial}{\partial x} \tau(x, y)\right| \geq C_{4} \text {, and }\left|\frac{\partial}{\partial y} \tau\left(x_{0}, y_{0}\right)\right|<C_{1} \text { for at least one }\left(x_{0}, y_{0}\right) \in K \text {. }
$$

2. Class V (vertical gradient): For all $(x, y) \in K$ we have 


$$
\left|\frac{\partial}{\partial y} \tau(x, y)\right| \geq C_{1} \text {, and }\left|\frac{\partial}{\partial x} \tau\left(x_{0}, y_{0}\right)\right|<C_{4} \text { for at least one }\left(x_{0}, y_{0}\right) \in K .
$$

3. Class D (diagonal gradient): For all $(x, y) \in K$ we have

$$
\left|\frac{\partial}{\partial x} \tau(x, y)\right| \geq C_{4} \text { and }\left|\frac{\partial}{\partial y} \tau(x, y)\right| \geq C_{1} .
$$

This is illustrated in Fig. 7. If a cell $K$ belongs to class $\mathrm{H}$ (resp. class V), then also all
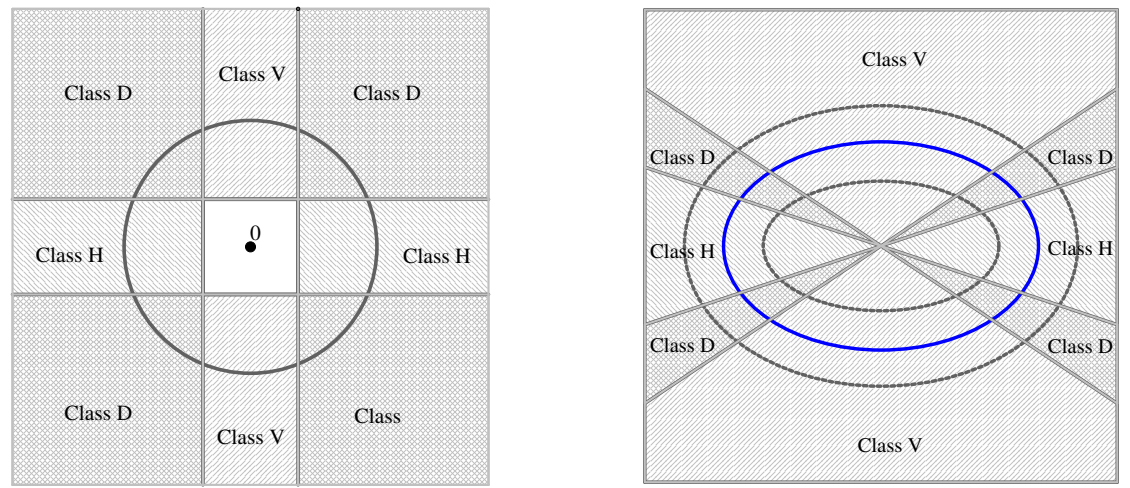

Fig. 7 Illustration of the regions of cell classes V, H and D. Left: The regions defined by $\nabla \tau(x, y)$ for $(x, y)$ over the union of the trimmed cells. The black circle has radius $4 C_{1}=$ $\min _{\tau(x, y)=0}\|\nabla \tau(x, y)\|_{2}$. It is visible that $\nabla \tau(x, y)$ lies outside the square in the middle. Right: An example trimming curve (an ellipse, shown in blue). The dotted offsets enclose the region that contains trimmed cells.

of its neighbors are either in class $\mathrm{H}$ (resp. V) or in class D. To obtain the modified subdivision $K^{\star}$ we merge all pairs of vertically adjacent cells where one of them is in class H. Similarly, we merge all pairs of horizontally adjacent cells where one of them is in class V. The remaining cells are kept. Note that this results in rectangular cells of maximum size $h$, since at most two cells will be merged, due to the restricted range of the gradients. The modified rule CLT ${ }^{\star}$ is obtained by applying CLT to the modified subdivision $\mathscr{K}^{\star}$.

Next, we prove the convergence result for the modified rule CLT ${ }^{\star}$. The modified quadrature cells in $\mathrm{CLT}^{\star}$ ensure that all triangular cells belong to class $\mathrm{D}$, where we have a bound on both partial derivatives, cf. (53). Moreover, all cells satisfy the assumptions about the base cases in the strong sense.

Theorem 1. Assume $\tau \in C^{2}\left([0,1]^{2}\right)$ such that the constant $C_{1}=C_{4}$ as defined in (50) is positive, and $f \in C^{4}\left([0,1]^{2}\right)$. Then, there exists a constant $C_{\tau, f}$, such that the $\mathrm{CLT}^{\star}$ rule with $n=2$ and $k=2$ satisfies

$$
\left|I_{\tau} f-\operatorname{CLT}^{\star}(h, 2,2) f\right| \leq C_{\tau, f} h^{3} .
$$


Proof. By the construction of $\mathrm{CLT}^{\star}$, the subdivision $\mathscr{K}^{\star}$ consists of untrimmed quadrature cells, trimmed quadrilateral cells of classes $\mathrm{H}, \mathrm{V}$ and $\mathrm{D}$, and trimmed triangular and pentagonal cells of class D. In the trimmed quadrilateral cells we can always apply Lemma 1, while in the trimmed triangular cells of class D we can apply Lemma 2. Moreover, we can treat the trimmed pentagonal cells of class D by applying Lemma 2 to the complement of the quadrature domain. In both cases the constants $C_{2}$ and $C_{3}$ are obtained by linear approximation of $\tau$. They depend on the second derivative of $\tau$ whose norm is bounded.

The number of trimmed cells does not exceed $C_{5} \frac{1}{h}$ for some constant $C_{5}$. Moreover, we can use the same constants $C_{\text {quad }}$ and $C_{\text {triangle }}$ for all trimmed cells. Indeed, these constants depend on the values $C_{1}, \ldots, C_{4}$, which are determined by derivatives of the trimming function. Consequently, a global upper bound for these constants exists and depends solely on the trimming function $\tau$. Moreover, we may use an upper bound on the derivatives of $f$. We conclude

$$
\sum_{K \in \mathscr{K}_{\text {trimmed }}^{\star}}\left|I_{\tau, K} f-\mathrm{CLT}_{K}(h, 2,2) f\right| \leq C_{5} \max \left\{C_{\text {quad }}, C_{\text {triangle }}\right\} h^{3} .
$$

Since we use $n=2$ Gauss nodes in each direction for the untrimmed cells, the local error is bounded by $C_{\text {Gauss }} h^{5}$ in each of these cells for some constant $C_{\text {Gauss }}$. Since there are at most $\frac{1}{h^{2}}$ untrimmed cells, we have

$$
\sum_{K \in \mathscr{K}_{\text {untrimmed }}^{\star}}\left|I_{\tau, K} f-\operatorname{CLT}_{K}(h, 2,2) f\right| \leq C_{\text {Gauss }} h^{3} .
$$

The result (54) is implied by these two inequalities, since the various constants depend on $\tau$ and $f$ only.

We conjecture that in the triangular base case the influence of the last term in (44) is canceled by the corresponding term in the adjacent cell. Consequently, in practice it suffices to use CLT instead of CLT ${ }^{\star}$. This is supported by our numerical experiments, where CLT is tested.

\section{Numerical experiments}

We implemented the method in C++ using the G+Smo library [17]. In this section, we will test the approximation properties of the linearized trimmed quadrature rule CLT as well as the linearized trimmed quadrature rule LT on a number of trimmed geometries. Implementing the modified rule CLT ${ }^{\star}$ that was constructed in Section 6 for the theoretical analysis can be computationally inefficient in practice since it is necessary to estimate the gradient of $\tau$ in all cells for deciding which cells are to be joined. Additionally, one needs to find an upper bound of the gradient's norm along the trimming curve. However, we also implemented CLT ${ }^{\star}$ in a particular case in order to make a direct comparison with CLT. In the numerical experiments we 
observe that the theoretical error estimate for CLT ${ }^{\star}$ (Theorem 1) still holds for the original CLT quadrature rule.

\subsection{Ellipse}

As a first example, we use our method to compute the volume of an ellipse implicitly defined by

$$
\tau(x, y)=-\frac{(x-0.5)^{2}}{a^{2}}-\frac{(y-0.5)^{2}}{b^{2}}+1>0,
$$

where we set $a=0.45$ and $b=0.2$ in our experiment. Fig. 8 shows the result of the subdivision of this ellipse after some steps of refinement. In each cell, the trimming function was approximated by a linear function as described in Section 4. Note that in the pentagonal case we integrate over the remaining triangle and subtract from the full integral. In the left plot in Fig. 9 we show the quadrature error for different val-

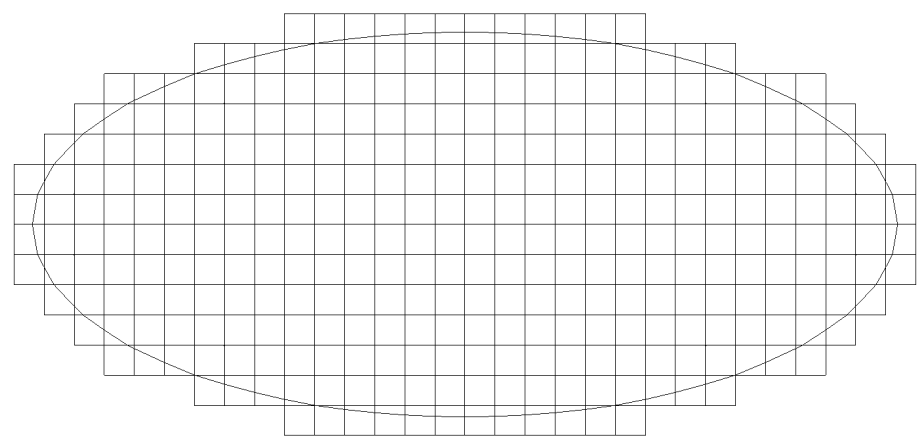

Fig. 8 Subdivision of the ellipse after some steps of refinement with approximate linear trimming curve in each cell.

ues of $h$ when computing the area enclosed by the ellipse with the simple trimmed quadrature $L T(h, 1)$ described in Section 4 and with the trimmed quadrature by first order correction $\operatorname{CLT}(h, 1,2)$ described in Section 5. We observe that the first order error correction results in an additional order of convergence with respect to $h$, confirming the theoretical result from Theorem 1.

Next, we show the computation times for both quadrature rules in Fig. 10. We observe that applying the error correction does not result in a significant increase in complexity compared to the linearized trimmed quadrature. The complexity for both LT and CLT increases linearly with the number of cells.

Finally, we compare the CLT quadrature rule with the modified quadrature rule $\mathrm{CLT}^{\star}$ which was defined in Section 6 for the theoretical analysis of the convergence. For the ellipse given by the trimming function (57) the exact value of the constants 


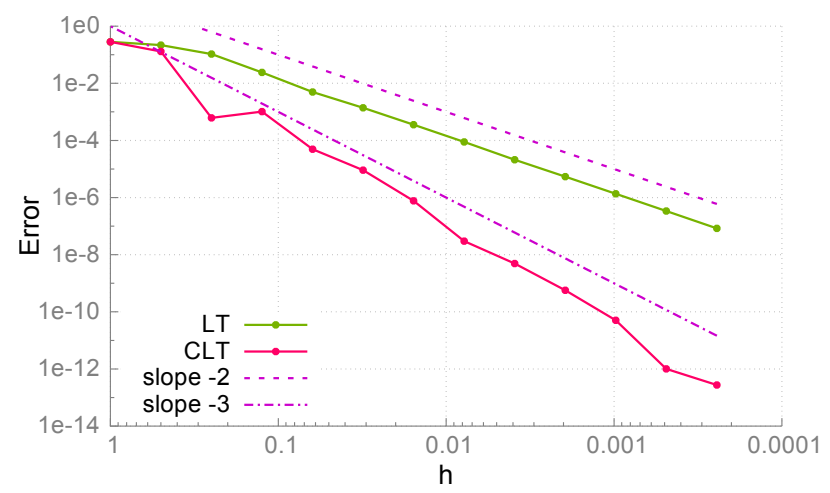

Fig. 9 Absolute error in LT and CLT for the area enclosed by the ellipse.

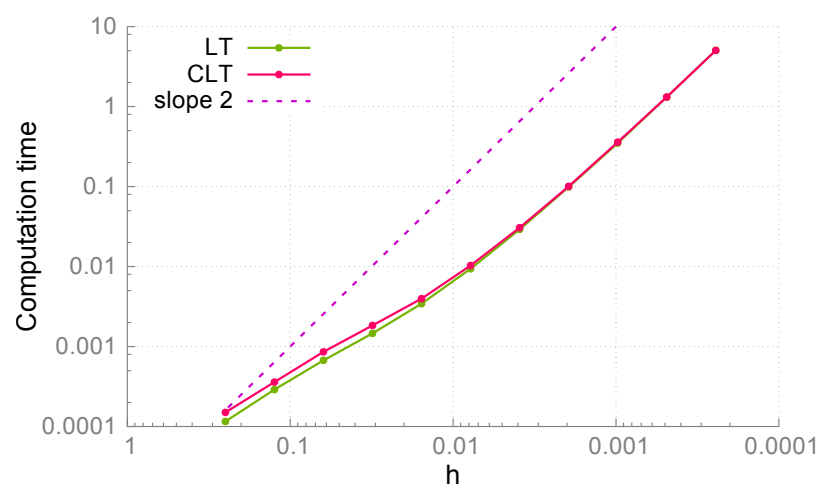

Fig. 10 Computation times for the approximation of the area enclosed by the ellipse

$C_{1}=C_{4}$ in the conditions (51), (52) and (53) is

$$
C_{1}=\frac{1}{4} \min _{\tau(x, y)=0}\|\nabla \tau(x, y)\|_{2}=\frac{10}{9} .
$$

We sample the gradient of $\tau$ on a fine grid in each cell in order to check these conditions. In Figure 11 we show the modified subdivision of the ellipse after some steps of refinement. Eight cells were joined in this case, they are highlighted in the picture. Figure 12 shows the error of both CLT and CLT ${ }^{\star}$ when computing the area of the ellipse. We observe that the quadrature errors for both methods have the same asymptotic behavior. 


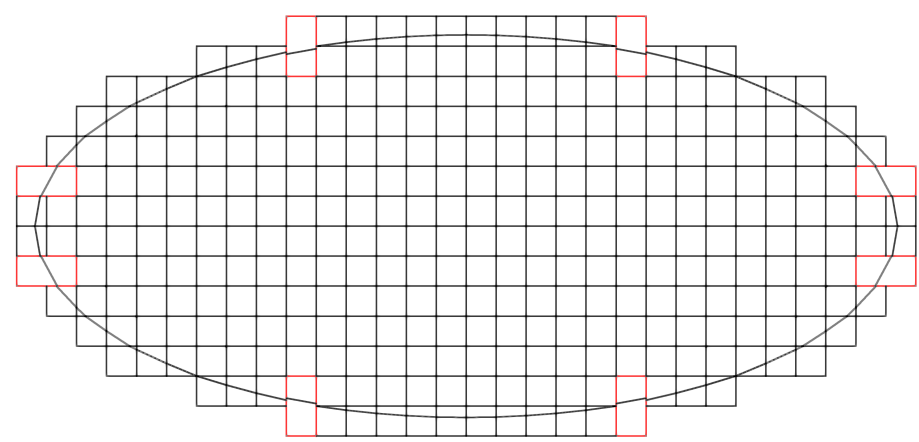

Fig. 11 Modified subdivision of the ellipse for CLT ${ }^{\star}$ after five steps of refinement. Joined cells are highlighted.

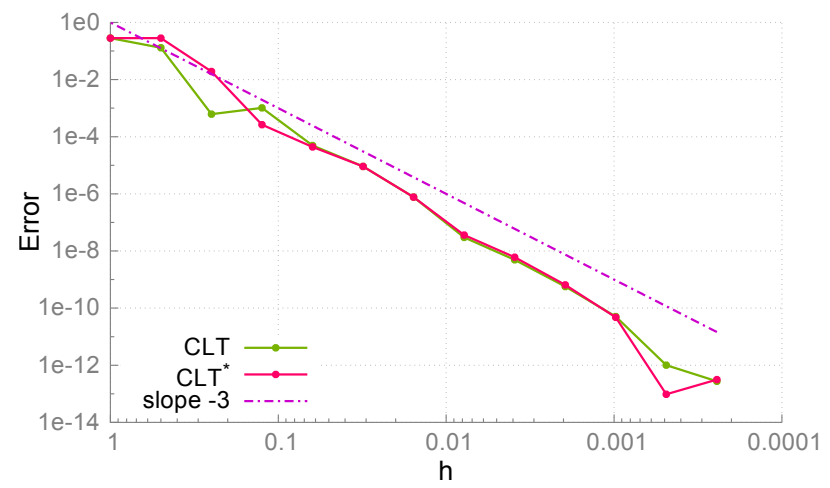

Fig. 12 Absolute error in CLT and CLT ${ }^{\star}$ for the area enclosed by the ellipse.

\subsection{Perforated quarter annulus}

In our next example, we will approximate the area of a quarter annulus which is trimmed with three circles. The domain is represented by Non-Uniform Rational BSplines (NURBS), which is a powerful technique for the representation of complex geometric shapes, see [22] for more details. The quarter annulus is represented exactly as a NURBS domain of polynomial degrees $\left(p_{1}, p_{2}\right)=(1,2)$ with no interior knots ${ }^{1}$. Fig. 13 shows the piecewise linear approximation of the trimming curve in the computational and in the parametric domain after some steps of refinement.

Since we perform the computation on the parametric domain, we approximate the integral

$$
\int_{\Omega_{\gamma^{\circ} G}}\left|\operatorname{det} J_{G}(x, y)\right| \mathrm{d} y \mathrm{~d} x,
$$

${ }^{1}$ The weights were chosen as $\left(\omega_{0,0}, \omega_{1,0}, \omega_{0,1}, \omega_{1,1}, \omega_{0,2}, \omega_{1,2}\right)=\left(1,1, \frac{1}{\sqrt{2}}, \frac{1}{\sqrt{2}}, 1,1\right)$ and the corresponding control points as $(1,0),(2,0),(1,1),(2,2),(0,1),(0,2)$. 

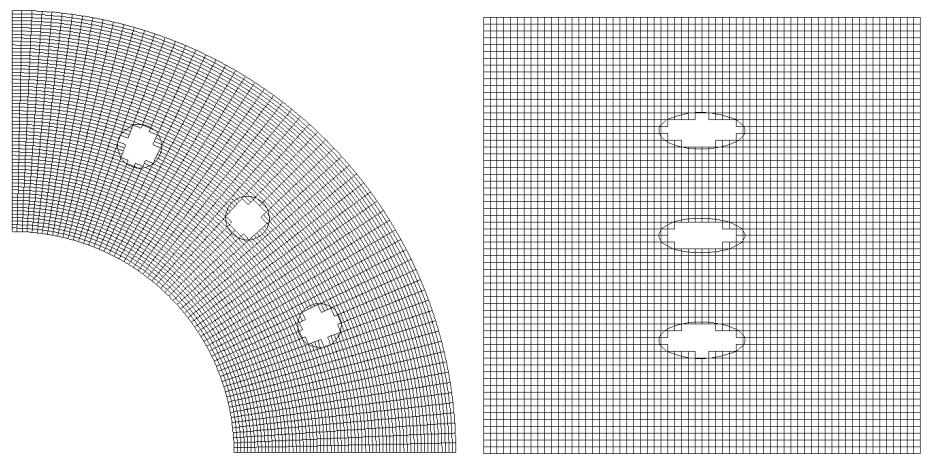

Fig. 13 Subdivision of the perforated quarter annulus and its corresponding parametric domain after some steps of refinement with approximate linear trimming curve in each cell.

where $G:[0.1]^{2} \rightarrow \mathbb{R}^{2}$ is the NURBS parameterization of the quarter annulus and $J_{G}(x, y)$ is the Jacobian matrix of $G$ at the point $(x, y)$. The trimming function $\gamma: \mathbb{R}^{2} \rightarrow \mathbb{R}$ on the physical domain is defined as the product of the implicit representations of the three circles.

In the first plot in Fig. 14 we show the convergence rate of the quadrature rules with and without error correction. We chose $n=2$ Gauss nodes for the linearized quadrature and $k=2$ Gauss nodes for the correction term. As in the case of the

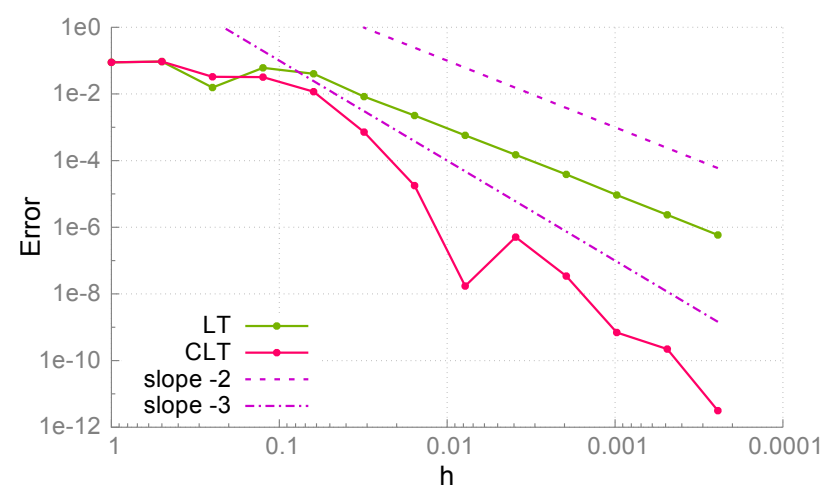

Fig. 14 Absolute error in LT and CLT for the area of the perforated quarter annulus for $n=2, k=2$.

ellipse, we observe that the first order error correction term in CLT results in an additional order of convergence compared to the linearized quadrature in LT.

Since we only use one error correction term, the convergence error cannot be improved by additional Gauss nodes in the bivariate and univariate quadrature. This 
is confirmed by the second plot in Fig. 15 which shows the same experiment as in the first plot but for $n=3$ and $k=3$.

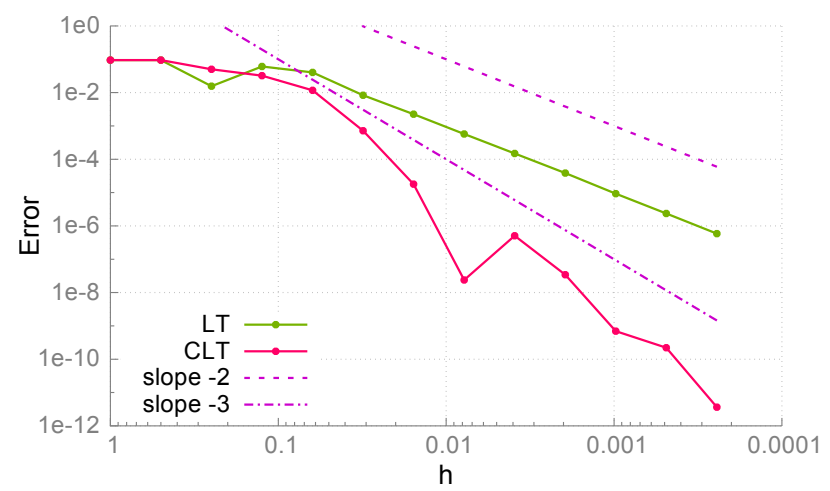

Fig. 15 Absolute error in LT and CLT for the area of the perforated quarter annulus for $n=3, k=3$.

\subsection{Singular case: Bicuspid curve}

Fig. 16 shows a linear approximation of the bicuspid curve which is an algebraic curve given by

$$
\left(x^{2}-a^{2}\right)(x-a)^{2}+\left(y^{2}-a^{2}\right)^{2}=0
$$

for some $a>0$. It has two cusps that hinder the improvement of the approximation order by the error correction term. In Fig. 17 we show the error convergence of the

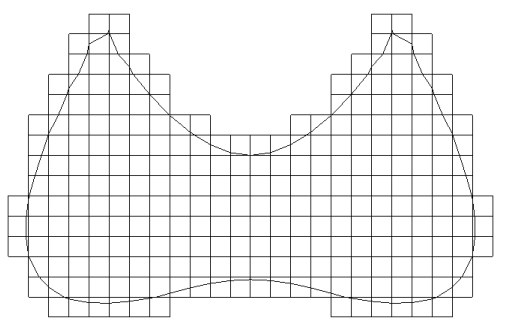

Fig. 16 Subdivision of the bicuspid with approximate linear trimming curve in each cell.

approximation of the area enclosed by the bicuspid, where the reference value was computed with a lower value of $h$. We observe that the error correction in CLT does not improve the convergence rate in this case, however, the absolute error is lower. 


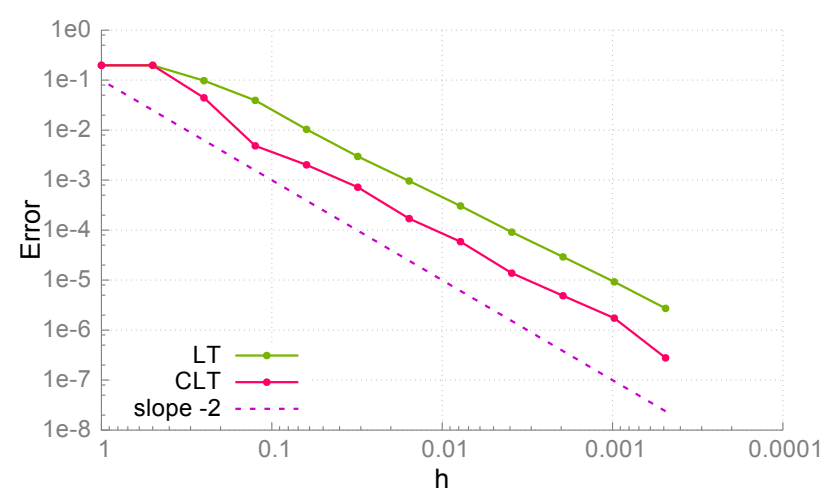

Fig. 17 Absolute error for the computation of the area enclosed by the bicuspid curve using LT and CLT.

\section{Conclusion}

We presented a novel method for quadrature on trimmed two-dimensional domains which was shown to be accurate, efficient and easy to implement. In particular, cubic convergence was both proved and observed in our experiments, while the computation times were not compromised.

Two generalizations are possible. On the one hand, more terms of the Taylor expansion (16) can be added to the result in order to further improve the order of convergence of the quadrature error. This is necessary for applying the method to isogeometric discretizations of higher approximation power. Our conjecture is that each term of the Taylor expansion raises the order of convergence by one.

On the other hand, the method has the potential to be generalized to threedimensional domains. We expect that the generalization will follow the same main ideas as in the two-dimensional case. However, the number of base cases will be higher.

Acknowledgements The authors gratefully acknowledge the support provided by the Austrian Science Fund (FWF) through project NFN S11708 and by the European Research Council (ERC), project GA 694515.

\section{References}

1. Bandara, K., Rüberg, T., Cirak, F.: Shape optimisation with multiresolution subdivision surfaces and immersed finite elements. Computer Methods in Applied Mechanics and Engineering 300(Supplement C), 510 - 539 (2016)

2. Bazilevs, Y., Calo, V., Cottrell, J., Evans, J., Hughes, T., Lipton, S., Scott, M., Sederberg, T.: Isogeometric analysis using T-splines. Computer Methods in Applied Mechanics and Engineering 199(5), 229 - 263 (2010) 
3. Beer, G., Marussig, B., Zechner, J.: A simple approach to the numerical simulation with trimmed CAD surfaces. Computer Methods in Applied Mechanics and Engineering 285, 776-790 (2015)

4. Bordas, S.P.A., Burman, E.N., Larson, M.G., Olshanskii, M.A. (eds.): Geometrically Unfitted Finite Element Methods and Applications. Lecture Notes in Computational Science and Engineering. Springer International Publishing (2017)

5. Burman, E., Hansbo, P., Larson, M.G.: A cut finite element method with boundary value correction. Mathematics of Computation 87, 633-657 (2018)

6. Cox, D.A., Sederberg, T.W., Chen, F.: The moving line ideal basis of planar rational curves. Computer Aided Geometric Design 15(8), 803 - 827 (1998)

7. Erik, B., Susanne, C., Peter, H., G., L.M., André, M.: CutFEM: Discretizing geometry and partial differential equations. International Journal for Numerical Methods in Engineering 104(7), 472-501

8. Gander, W., Gautschi, W.: Adaptive quadrature-revisited. BIT Numerical Mathematics 40(1), 84-101 (2000)

9. Hofer, C., Langer, U., Toulopoulos, I.: Discontinuous Galerkin isogeometric analysis of elliptic diffusion problems on segmentations with gaps. SIAM Journal on Scientific Computing 38(6), A3430-A3460 (2016)

10. Hofer, C., Toulopoulos, I.: Discontinuous Galerkin isogeometric analysis for parametrizations with overlapping regions. RICAM-Report 2017-17 (2017)

11. Hughes, T., Cottrell, J., Bazilevs, Y.: Isogeometric analysis: CAD, finite elements, NURBS, exact geometry and mesh refinement. Computer Methods in Applied Mechanics and Engineering 194(39-41), 4135-4195 (2005)

12. Jüttler, B., Mantzaflaris, A., Perl, R., Rumpf, M.: On numerical integration in isogeometric subdivision methods for PDEs on surfaces. Computer Methods in Applied Mechanics and Engineering 302, 131 - 146 (2016)

13. Kamensky, D., Hsu, M.C., Schillinger, D., Evans, J.A., Aggarwal, A., Bazilevs, Y., Sacks, M.S., Hughes, T.J.: An immersogeometric variational framework for fluid-structure interaction: Application to bioprosthetic heart valves. Computer Methods in Applied Mechanics and Engineering 284, 1005 - 1053 (2015). Isogeometric Analysis Special Issue

14. Kim, H., Seo, Y., Youn, S..: Isogeometric analysis for trimmed CAD surfaces. Computer Methods in Applied Mechanics and Engineering 198(37-40), 2982-2995 (2009)

15. Kumar, S., Manocha, D.: Efficient rendering of trimmed nurbs surfaces. Computer-Aided Design 27(7), 509 - 521 (1995). Display and visualisation

16. Lehrenfeld, C.: High order unfitted finite element methods on level set domains using isoparametric mappings. Computer Methods in Applied Mechanics and Engineering 300, 716 - 733 (2016)

17. Mantzaflaris, A., Scholz, F., others (see website): G+Smo (Geometry plus Simulation modules) v0.8.1. http://gs.jku.at/gismo (2018)

18. Marussig, B., Hiemstra, R., Hughes, T.: Improved conditioning of isogeometric analysis matrices for trimmed geometries. Computer Methods in Applied Mechanics and Engineering 334, 79-110 (2018)

19. Marussig, B., Hughes, T.: A review of trimming in isogeometric analysis: Challenges, data exchange and simulation aspects. Archives of Computational Methods in Engineering pp. 1-69 (2017)

20. Nagy, A., Benson, D.: On the numerical integration of trimmed isogeometric elements. Computer Methods in Applied Mechanics and Engineering 284, 165-185 (2015)

21. Newman, T.S., Yi, H.: A survey of the marching cubes algorithm. Computers \& Graphics 30(5), $854-879$ (2006)

22. Pigl, L.A., Tiller, W.: The NURBS book. Springer-Verlag Berlin Heidelberg (1997)

23. Rüberg, T., Cirak, F.: A fixed-grid b-spline finite element technique for fluid-structure interaction. International Journal for Numerical Methods in Fluids 74(9), 623-660 (2014)

24. Ruess, M., Schillinger, D., Özcan, A., Rank, E.: Weak coupling for isogeometric analysis of non-matching and trimmed multi-patch geometries. Computer Methods in Applied Mechanics and Engineering 269, 46-71 (2014) 
25. Sanches, R., Bornemann, P., Cirak, F.: Immersed b-spline (i-spline) finite element method for geometrically complex domains. Computer Methods in Applied Mechanics and Engineering 200(13), 1432 - 1445 (2011)

26. Schmidt, R., Wüchner, R., Bletzinger, K.U.: Isogeometric analysis of trimmed NURBS geometries. Computer Methods in Applied Mechanics and Engineering 241-244, 93-111 (2012)

27. Scholz, F., Mantzaflaris, A., Jüttler, B.: Partial tensor decomposition for decoupling isogeometric Galerkin discretizations. Computer Methods in Applied Mechanics and Engineering 336, 485 - 506 (2018)

28. Seiler, A., Jüttler, B.: Reparameterization and adaptive quadrature for the isogeometric discontinuous Galerkin method. In: Mathematical Methods for Curves and Surfaces, pp. 251-269. Springer International Publishing, Cham (2017)

29. Seo, Y.D., Kim, H.J., Youn, S.K.: Isogeometric topology optimization using trimmed spline surfaces. Computer Methods in Applied Mechanics and Engineering 199(49-52), 3270-3296 (2010)

30. Taubin, G.: Estimation of planar curves, surfaces, and nonplanar space curves defined by implicit equations with applications to edge and range image segmentation. IEEE Transactions on Pattern Analysis and Machine Intelligence 13(11), 1115-1138 (1991)

31. Zhang, H., Mo, R., Wan, N.: An IGA discontinuous Galerkin method on the union of overlapped patches. Computer Methods in Applied Mechanics and Engineering 326, 446-480 (2017)

32. Zhu, X., Ma, Z., Hu, P.: Nonconforming isogeometric analysis for trimmed CAD geometries using finite-element tearing and interconnecting algorithm. Proceedings of the Institution of Mechanical Engineers, Part C: Journal of Mechanical Engineering Science 231(8), 1371-1389 (2017) 